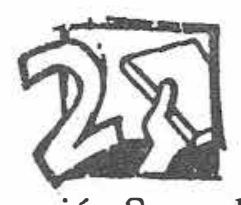

http://doi.org/10.15359/ree.2004-7.12

Educación Secundaria

\title{
INTEGRACIÓN DEL ASPECTO CULTURAL EN LA CLASE DE INGLÉS
}

Sonia Calderón

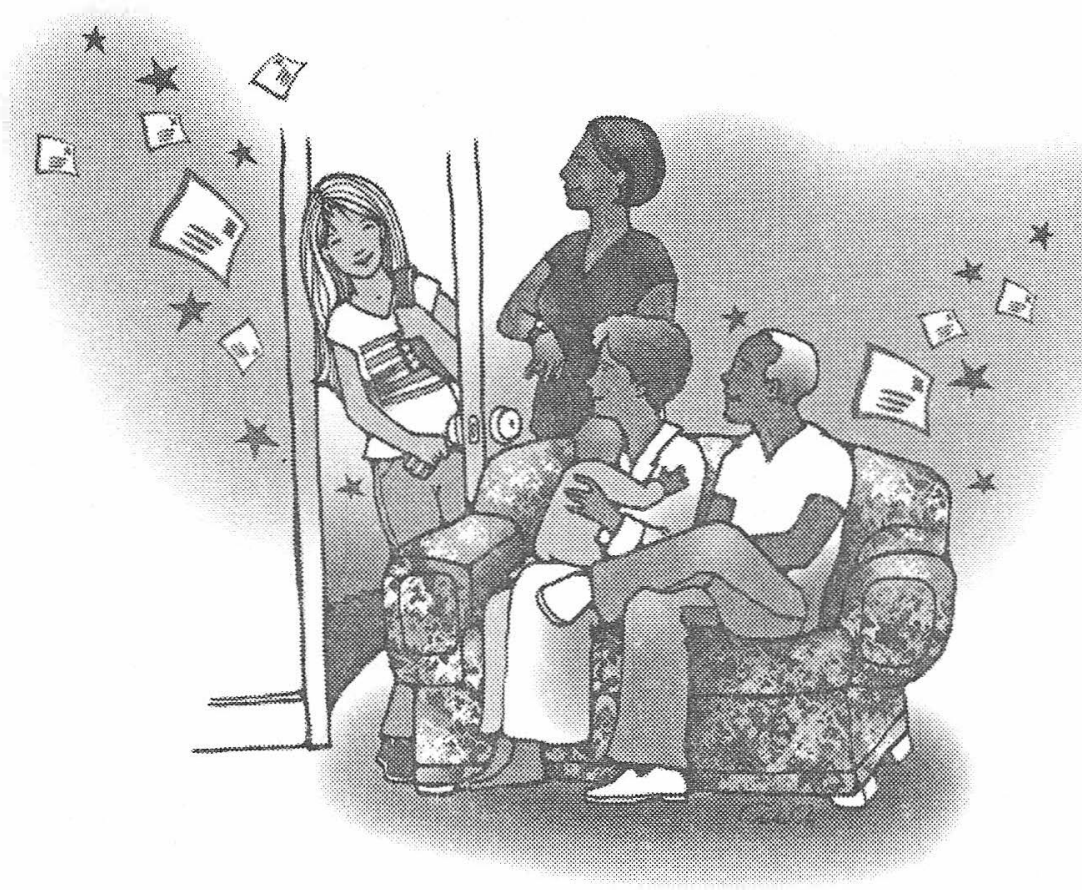

* Tiene un bachillerato y un profesorado en Inglés de la Universidad de Costa Rica y una licenciatura en Lingüística Aplicada de la Universidad Nacional. Además, completó cursos de postgrado en Lingüística en la Universidad de Georgetown, Washington D. C. en los estados Unidos. Trabajó como docente en el área de inglés en la Escuela de Literatura y Ciencias del Lenguaje de la Universidad Nacional desde 1991 hasta el 2002 y desde enero del 2004 hasta el presente. También tiene experiencia como docente y coordinadora de programas de capacitación y desarrollo curricular del Centro Cultural Costarricense Norteamericano. Sus principales áreas de interés académico son la lingüística aplicada, la enseñanza de la gramática y la evaluación de los aprendizajes. 
Nada tiene más poder que la costumbre o el hábito.

Ovidio

\section{Introducción}

El hombre actual vive en un mundo que se hace cada vez más pequeño debido a los medios de comunicación masiva. No importa el campo en el que el ser humano se desempeñe, el contacto con personas pertenecientes a otras culturas es inevitable. En la Costa Rica de hoy en día, los hombres y mujeres no necesitan salir de las fronteras de su países para tener experiencias interculturales, muchos extranjeros viven en este país y tienen costumbres y hábitos distintos a los de los costarricenses.

El campo de estudio de la comunicación intercultural es relativamente nuevo. Durante la época colonial se perseguía la asimilación de los indígenas a la cultura de la potencia colonizadora. En el siglo XX y después de la Segunda Guerra Mundial, la creación de la Organización de Naciones Unidas determinó la necesidad de comunicación de los distintos pueblos y culturas. El gobiemo de los Estados Unidos enviaba diplomáticos a diferentes países y los capacitaba en el idioma del lugar. Dichos representantes solían tener problemas de comunicación, pues conocían el lenguaje, pero carecían de la habilidades interculturales necesarias para tener una experiencia satisfactoria. El antropólogo estadounidense Edward T. Hall se interesó en el tema y después de su experiencia de observación con la cultura Hopi, escribió el libro El lenguaje silencioso (1959), el cual se convirtió en la piedra angular del campo de la comunicación intercultural.

En los años setentas en los Estados Unidos la comunicación intercultural se fue consolidando como una disciplina académica y a principios de esa década se fundó la Asociación Internacional para la Comunicación Intercultural (SIETAR), la cual promueve la educación y la difusión de esta disciplina por medio de simposios, seminarios y publicaciones. Además de la asociación internacional, existen otras regionales, por ejemplo, en Europa. La comunicación intercultural y el desarrollo de habilidades para una adecuada interacción han sido exhaustivamente estudiadas desde mediados del siglo XX. Esa nueva concepción de la cultura se puede aplicar en muchas disciplinas: la diplomacia, las 
Since culture and language are closely related, culture cannot be left aside when teaching languages. Cultural training is much more than being exposed to cultural information; it includes the critical evaluation of such information and a comparison with one's culture. In today's globalized world, cultural competence has become fundamental. The following article introduces a unit to incorporate culture into the English class as a foreign language in Costa Rica. It suggests activities to develop intercultural awareness and linguistic objectives. relaciones internacionales, la sociología, la política internacional, la educación y la enseñanza de idiomas extranjeros. Los expertos observan y analizan el comportamiento humano de grupos específicos y sacan conclusiones sobre sus valores, hábitos y comportamiento. Cuestiones como la distancia personal y el lenguaje corporal son de extrema importancia al interactuar como miembros de otros ámbitos culturales.

En el campo de la adquisición de una segunda lengua, John Schumann propuso el modelo de aculturación que explica que el grado de competencia del individuo al aprender un segundo idioma depende de su nivel de aculturación. Se debe tomar en cuenta la relación entre la cultura madre y la cultura meta, la congruencia de la cultura madre y la opinión de los miembros de la cultura meta sobre la cultura madre. A pesar de que actualmente el modelo de aculturación de Schumann no es aceptado por muchas linguiistas, sus ideas han tenido gran influencia en el campo de la enseñanza de idiomas extranjeros. No obstante de que dicho modelo se refiere al aprendizaje de un idioma en el entorno donde ese idioma es la lengua nativa, se puede decir que en el ámbito de una lengua extranjera, que es el caso del inglés en Costa Rica, también se da cierto proceso de aculturación.

El desarrollo de la competencia intercultural va más allá de proveer a los estudiantes con información sobre la cultura meta. Aunque técnicas, tales como la "cápsula cultural" y la "isla cultural" pueden usarse en el aula. La cápsula cultural consiste en dedicar un rincón del aula a un tema sobre la cultura meta y colocar ahí cuadros, afiches, objetos referentes a ese tema, por ejemplo, el Halloween o día de brujas tendría a Jack O'lantern (la calabaza), la frase trick or treat (trato o truco), algunos disfraces y confites. Sin embargo, el entendimiento intercultural va más allá: el individuo debe desarrollar habilidades para la comprensión de la cultura meta, tales como la empatía y la permeabilidad del ego.

Los antropólogos han hecho varias clasificaciones de las culturas de acuerdo con sus características más salientes. Uno de los aspectos más interesantes en la comunicación intercultural es cómo el significado del concepto del tiempo cambia en diferentes pueblos y cómo todo el quehacer diario de la gente se ve modificado por este. En relación con este tema, las culturas se pueden clasificar como monocrónicas y policrónicas. 
American time is what I have termed "monochronic"

that is, Americans, when they are serious, usually prefer

to do one thing at a time, and this requires some kind

of scheduling, either implicit or explicit.

$P$-time (polychronic) sustems are characterized by

several things happening at once. They stress involve-

ment kof people rather than adhkerence to preset schedules. 1

(Hall, 1976:17).

Las culturas monocrónicas planean sus actividades, se rigen por horarios, proponen objetivos, hacen una labor a la vez y evitan las interrupciones. Sus fechas límite realmente lo son y separan su vida pública y privada. Las personas de las culturas policrónicas se ven influenciada por su entorno; tratan de hacer una labor a la vez, sus fechas límite son flexibles y les gusta compartir su vida privada con compañeros de trabajo. La cultura americana se clasifica como monocrónica y la cultura costarricense como policrónica.

En el campo de la enseñanza y aprendizaje de un idioma extranjero, el componente intercultural no ha recibido la atención necesaria. Si se analizan cuidadosamente varios libros de texto de los usados en el país para el aprendizaje del inglés como lengua extranjera, se puede notar que dichos recursos contienen poca imformación de la cultura meta o bien incluyen información esterotipada o carecen por completo de dicho componente. Son ejemplos de estos los textos Learn English diseñados por el Ministerio de Educación Pública para la Educación Secundaria. Además, un texto muy usado a nivel universitario y parauniversitario, la serie $\mathrm{New}$ Interchange (Cambridge University Press, 1996) contiene información escasa y no pertinente de la cultura meta. Otro ejemplo es el libro On Targest 1 (Scott, Foresman, 1991) que presenta una imagen estereotipada de la cultura norteamericana.

Las nociones de lengua y de cultura conforman un ente indisoluble. El lenguaje es más que la síntaxis, la morfología y el léxico. Un individuo debe adquirir competencia comunicativa en el segundo idioma: la habilidad de mantener una conversación. Además de la competencia meramente lingưística, la persona debe tener competencia sociolingüística y competencia sociopragmática: la habilidad de escoger el registro adecuado, según el contexto y sus interlocutores y la capacidad de realizar distintos actos del habla con el lenguaje. El propósito fundamental de un idioma es que los seres humanos se puedan comunicar. Tal y como lo dice el lingüista Peter Trudgill (198: 2), el lenguaje sirve para establecer relaciones sociales y proveer información sobre el que habla: 
These two aspects of language behaviour are very important from a social point of view: first, the function of language in establishing social relationships; and, second, the role played by language in conveying information about the speaker.

De ahí la importancia de incluir, de manera sistemática el contenido cultural y el análisis tanto de la cultura propia como de la cultura extranjera en el campo de la enseñanza de otros idiomas.

La propuesta siguiente consiste no solo en informar a los educandos de que existen diferencias entre los hábitos y valores de las culturas, si no de cómo dichas diferencias pueden afectar las relaciones interpersonales. Además, se pretende que los estudiantes puedan reflexionar sobre su propia cultura y ver esta con ojos críticos.

\section{¿Por qué una unidad sobre el concepto del tiempo?}

El adquirir conciencia sobre las diferencias interculturales y el desarrollo de habilidades para el trato intercultural no son solo competencia del estudiante de un idioma extranjero. Debido a los avances en la tecnología, nuestro mundo se ha vuelto más pequeño. Los abogados, ingenieros, educadores y médicos deben comunicarse con colegas de diversas culturales. Por teléfono, por escrito o por medios electrónicos.

Muchos malentendidos se deben a que la gente de diversas culturas tiene un concepto diferente del tiempo y su significado: lo que es perfectamente aceptable en un país puede ser ofensivo en otro. Los negocios pueden fallar y las relaciones personales pueden no tener ocasión de desarrollarse. Como seres culturales, tendremos a ser etnocéntricos, por lo que el papel de la capacitación intercultural radica en eliminar esa actitud.

Los patrones culturales se entretejen en una fina pieza de tela y ayudan a satisfacer las necesidades básicas de los individuos. El objetivo final de esta unidad es que los estudiantes adquieran conciencia de las diferencias culturales. Asimismo, la unidad tendrá tanto objetivos culturales como objetivos lingüísticos.

\section{Objetivos culturales}

1. Enunciar las diferencias de comportamiento en relación con el tiempo.

2. Expresarse sobre los patrones de comportamiento de la Cultura 1 y de la Cultura 2. 
3. Tomar conciencia de prácticas negativas como estereotipar y hacer comentarios etnocéntricos.

4. Poder explicar en forma oral y escrita por qué los malentendidos ocurren en la comunicación con gente de otras culturas.

\section{Objetivos lingüísticos}

1. Usar expresiones de contraste y comparación.

2. Dar una opinión.

3. Demostrar desacuerdo.

4. Hablar de hábitos y actividades diarias.

5. Hablar acerca de un vídeo.

6. Usar formas de reporte indirecto.

7. Familiarizarse con vocabulario específico del tema.

\section{Actividades de aprendizaje}

Para llevar a cabo la consecución de los objetivos se harán diversas actividades de aprendizaje que se detallan a continuación:

1. Proyección de un vídeo.

2. Preguntas de comprensión sobre el segmento de un vídeo.

3. Presentación sobre el tema del tiempo

4. Ejercicio de clarificación: la cultura madre.

5. Técnica de comparación: la cultura madre y la cultura meta.

6. Ejercicio de clarificación de valores.

7. Entrevista con una persona de la cultura meta.

8. Incidentes críticos.

9. Evaluación de la unidad.

10. Seguimiento de los objetivos lingüísticos.

\section{Primera sesión}

En la primera sesión los estudiantes observarán el comportamiento humano en un contexto intercultural. Esto se relaciona con el objetivo general de Sealye (1996: 31):

"La gente se comporta en cierta forma porque usan opciones que la sociedad les permite para llenar sus necesidades físicas y sicológicas básicas y los patrones culturales están interrelacionados y tienden a apoyar la satisfacción de las necesidades". 
Los participantes podrán describir el comportamiento evidenciado en el segmento de una forma objetiva y tomarán conciencia de por qué las relaciones interculturales pueden crear frustración.

El vídeo trata de un hombre de negocios americano que viaja al Medio Oriente para establecer un proyecto. Después de varios días, se empieza a sentir frustrado porque no puede hacer que el trabajo empiece: sus contactos locales continúan posponiendo el trabajo y parecen más interesados en reuniones sociales.

Los estudiantes ven el segmento de treinta minutos y después comentan los problemas que tuvo el hombre de negocios norteamericano y por qué los miembros de cada cultura tenía expectativas diferentes: los árabes estaban más interesados en establecer una relación social, primero, y después dedicarse a los negocios. El objetivo primordial del norteamericano era hacer que el trabajo se llevara a cabo lo más pronto posible.

Después de terminar la discusión del vídeo, los estudiantes hacen el siguiente ejercicio de clarificación de valores. Deben leer las oraciones y argumentar si están de acuerdo o en desacuerdo.

\section{Los costarricenses...}

1. Llegan tarde al trabajo.

2. Llegan tarde a clases.

3. Llegan tarde a fiestas.

4. Tienen por lo menos una hora de almuerzo.

5. Realizan más de una labor a la vez.

6. Pagan sus cuentas a tiempo.

El primer paso en el entendimiento intercultural radica en adquirir conciencia de la propia cultura. Las costumbres propias se deben ver con ojo crítico, lo que no resulta fácil, pues implica una transformación de la capacidad perceptiva del estudiante. La discusión de las oraciones propuestas en el ejercicio anterior permitirá a los participantes adquirir conciencia de sus hábitos propios, con los cuales los miembros de otras culturas tal vez no estén de acuerdo.

\section{Segunda sesión}

Los objetivos de la segunda sesión persiguen familiarizarse con diferentes significados de la idea de tiempo y describir el comportamiento de manera objetiva. Se usa la técnica de comparación por medio del siguiente cuadro: 


\begin{tabular}{|l|l|}
\hline \multicolumn{1}{|c|}{ Cultura madre } & \multicolumn{1}{c|}{ Cultura meta } \\
\hline $\begin{array}{l}\text { Las reuniones son extensas, de } \\
\text { varias horas }\end{array}$ & Las reuniones son cortas \\
$\begin{array}{l}\text { La agenda se rompe con } \\
\text { frecuencia }\end{array}$ & $\begin{array}{l}\text { Los temas se limitan a la } \\
\text { agenda }\end{array}$ \\
$\begin{array}{l}\text { Las reuniones se llevan a cabo } \\
\text { en horas de oficina }\end{array}$ & $\begin{array}{l}\text { Las reuniones se pueden hacer } \\
\text { en la hora del almuerzo }\end{array}$ \\
$\begin{array}{l}\text { Con frecuencia, muchas cosas } \\
\text { quedan sin resolver }\end{array}$ & $\begin{array}{l}\text { Muchos problemas se } \\
\text { resuelven }\end{array}$ \\
\hline
\end{tabular}

Después de analizar la información del Cuadro 2, los estudiantes tienen la oportunidad de trabajar con los siguientes incidentes críticos, los cuales son otra técnica usada en el desarrollo de habilidades interculturales. Ellos leen lo siguiente y contestan si es Falso o Verdadero.

1. Usted fue invitado por un amigo a una fiesta a las 8 p.m. Usted puede llegar a las 8:30 p.m.

2. Usted trabaja en una dependencia del estado. Su horario comienza a las 7:30 a.m. El reglamento de la institución establece que los empleados pueden llegar diez minutos tarde sin recibir ninguna reprimenda.

3. Un profesor le dio una asignación para presentar el día lunes. Usted no la hizo y le pregunta a él si la puede traer para la siguiente clase. El profesor acepta.

4. Yo tengo un horario para organizar mi trabajo y mis horas de estudio.

5. Para obtener una licencia de conducir o una renovación es necesario contar con al menos mediodía.

Para llevar a cabo el ejercicio descrito anteriormente, los estudiantes deben poder ver su cultura desde otro punto de vista.

\section{Tercera sesión}

Después de haber hecho dos ejercicios en el aula, ahora los participantes deben recurrir a trabajo de campo para recoger información sobre hábitos de un 
extranjero relacionados con el concepto del tiempo. Muchos extranjeros trabajan y viven en Costa Rica y debemos relacionamos con ellos por motivos laborales o personales.

Los estudiantes usarán la técnica de entrevistar a un representante de la cultura meta que ellos conozcan. Podrán usar el siguiente cuestionario como base y ampliar la información obtenida.

1. ¿Ha notado alguna diferencia entre el ritmo de vidaen Costa Rica y su país natal?

2. ¿Existe alguna diferencia en relación con las llegadas tardías?

3. ¿Se ha sentido frustrado en alguna situación social en relación con el tiempo (hora de llegada, hora de salida)?

4. ¿Cómo se comparan los horarios de transporte público en su país y en Costa Rica?

5. Otros comentarios.

\section{Cuarta sesión}

En la clase siguiente, los estudiantes se dividirán en grupos y compartirán la información recogido. Probablemente las respuestas obtenidas sean muy similares. Después de realizar el trabajo de campo de la tercera sesión y discutir la información obtenida, los estudiantes analizarán una serie de incidentes críticos. El objetivo de esta sesión es el de reflexionar sobre por qué las personas en estos casos se sintieron frustradas o molestas. A continuación la serie de incidentes con que los participantes trabajarán.

1. Una pareja de canadienses que viven en Costa Rica invitan a unos ticos a cenar el sábado por la noche a las 8:00 p.m. Algunos de los invitados llegan a las 8:20; otros llegan a las 8:45 y los últimos llegan a las 9:10. La anfiriona está un poco molesta, porque el asado en el horno se cocinó de más y la ensalada no está muy fresca. Los invitados no dan ninguna explicación por su hora de llegada.

2. Bill es un extranjero que vive en Costa Rica. Él solicita una cita con un médico en una clínica privada y la secretaria le da la cita para las 3:30 p.m. El llega a las 3:20. Hay otros pacientes en la sala de espera. El doctor llega a las cuatro y ve a otros pacientes de primero. Bill espera y espera. Finalmente a las 5:15 abandona la oficina de muy mal humor.

3. Los bancos del estado abren de 8:30 a.m. a 3:30 p.m., jornada continua. Sally, una inglesa, va a la sucursal del banco en Tibás a las 12:20. Hay una larga fila y a pesar de que hay cinco ventanillas en el banco, solo dos cajeros atienden. Es su hora de almuerzo. Sally debe esperar durante más de una hora. 
4. Tom es un extranjero que vive en Costa Rica desde hace dos años. Acaba de comprar una casa y llama a un electricista para que haga algunas reparaciones. El electricista está de acuerdo en llegar al día siguiente a las 9:00 a.m. Tom espera y espera. A la una de la tarde, Tom decide salir a hacer unas compras. Cuando regresa a las 4:00, hay una nota del electricista diciendo que llegó a las 3:15.

\section{Quinta sesión}

En esta sesión se trabajará en grupos pequeños, en los cuales los estudiantes deben comentar el significado de los siguientes refranes y relacionarlo con lo tratado en las sesiones anteriores:
A la larga, todo se arregla.
El que corre mucho, se cae de panza, y el que no corre no alcanza.
Quien a su tiempo descansa, rinde el doble y no se cansa.
Quien va pasito a pasito, llega descansado.
Más vale tarde que nunca.
Nunca es tarde si la dicha es buena.
No por mucho madrugar amanece más temprano.

\section{Sexta sesión}

Esta sesión enfocará los objetivos lingüísticos de la unidad. Consiste en varias actividades en las cuales los estudiantes aplicarán su conocimiento del léxico del idioma extranjero, su habilidad sociolingüística y su capacidad de expresión oral.

Los estudiantes explican el significado de los siguientes términos en inglés.

1. schedule (horario)

2. to be on time (llegar a la hora indicada)

3. to be late (llegar tarde)

4. coffee break (hora del café)

5. business hours (horas de negocios)

6. rush hour (hora pico)

7. lunch break (hora de almuerzo)

8. 24/7 (veinte y cuatro horas al día, siete días a la semana)

9. around the clock (todo el tiempo)

10. "tico time" (hora tica)

11. office hours (horas de oficina) 
Repaso de la función de mostrar acuerdo o desacuerdo.

1. Todos los negocios deberían estar abiertos a la hora de almuerzo.

2. Los bancos estatales deben abrir los sábados por la mañana.

3. Las oficinas de la universidad deberían atender a la hora de almuerzo.

4. Es importante hacer un horario de estudio y apegarse a él.

\section{Actividad 3:}

En forma individual, los estudiantes contestan Falso o Verdadero.

1. El concepto del tiempo es el mismo alrededor del mundo.

2. El ritmo de vida en la ciudad de San Josées más rápido que en Nicoya, Guanacaste.

3. Cuando interactuamos con extranjeros, pueden existir malentendidos debido a diferentes conceptos del tiempo.

4. Aún si nunca se vive en el extranjero, es importante estar conscientes y entender las diferencias culturales.

5. Nuestras costumbres son mejores que las de otros países.

\section{Conclusión}

La comunicación intercultural no se aplica solamente al campo de la enseñanza de idiomas extranjeros o a la lingüística, sino que una disciplina que cubre muchos otros campos, tales como las relaciones internacionales, la diplomacia, los negocios y las leyes. La incorporación de temas culturales en la clase de inglés debe ir más allá de proveer información sobre la cultura meta, debe buscar el desarrollo de destrezas para una efectiva interacción intercultural. El aprendizaje de un idioma extranjero no puede darse si no se toma en cuenta la cultura meta. Aprender un idioma es más que saber gramática, léxico y fonología; incluye el desarrollo de la competencia cultural.

La unidad descrita arriba cumple con objetivos culturales y lingüísticos y se puede llevar a cabo en una situación en donde los estudiantes tengan un nivel intermedio del inglés. Además de tener objetivos meramente culturales, las actividades promueven la comunicación en el aula por medio de trabajo en parejas, trabajo en grupos, aprendizaje cooperativo y ejercicios de clarificación de valores. Los estudiantes no solo aprenderán sobre la cultura del idioma que estudian, sino que también tendrán la oportunidad de reflexionar sobre su propia cultura. 


\section{Referencias}

Crawford-Lange, L. y Lange, D. (1984). Doing the Unthinkable in the Second Language Classroom. ACTFL Foreing Language Education Series. 15.

Fiber Luce, L. y Smith, E. eds. (1994). Toward Internationalism: Readings in Cross Cultural Communication. Nueva York Newbury House Publishers.

Gudykunst, W. B. y Ting Tooney, S. (1988). Culture and Interpersonal Communication. London: Sage Publishers.

Hall, E. T. 1976. Beyond Culture. Nueva York: Anchor Books.

Kim, Y. K. (1984). Communication and Acculturation Communicating Interculturally.

Martín, J. M. (2000). La interculturalidad: aspectos de su aprendizaje y educación desde la perspectiva de la interacción comunicativa. http://www.ub.es/ filhis/culturele/morillas.html.

Ruben, B. et al. (1977). Cross Cultural Effectiveness. Intercultural Communication: State of the Art. Pittsburg: SIETAR.

Seelye, N., ed. (1966). Experimential Activities for Intercultural Learning. Yarmouth, Maine: Intercultural Press.

Trudgill, P. (1983). Sociolinguistics: An Introduction to Language and Society. Middleses: Penguin Books.

Widdowson, H. (1988). Aspects of the relationship between culture and Language. Culture and Language. 